\title{
Medial prefrontal cortex deficits correlate with unrefreshing sleep in patients with Chronic Fatigue Syndrome
}

Zack Y. Shan ${ }^{1 *}$, Richard Kwiatek ${ }^{2}$, Richard Burnet $^{3}$, Peter Del Fante ${ }^{4}$, Donald R. Staines ${ }^{1}$, Sonya M. Marshall-Gradisnik ${ }^{1}$, Leighton R. Barnden ${ }^{1}$

${ }^{1}$ National Centre for Neuroimmunology and Emerging Diseases, Menzies Health Institute Queensland, Griffith University, Gold Coast, QLD, Australia

${ }^{2}$ Division of Medical Subspecialities, Lyell McEwin Hospital, Elizabeth Vale, SA, Australia ${ }^{3}$ Endocrinology department, Royal Adelaide Hospital, Adelaide, SA, Australia ${ }^{4}$ Healthfirst Network, Woodville, SA, Australia

Running Title: Brain structure and unrefreshing sleep in CFS

Word count: 6124

*Corresponding author: Zack Shan, Ph. D.; National Centre for Neuroimmunology and Emerging Diseases, Menzies Health Institute Queensland, Griffith University, Gold Coast, QLD 4222, Australia; T: +61 (7) 56780725, F: +61 (7) 56728908; E: z.shan@griffith.edu.au 


\section{Abstract}

Unrefreshing sleep is a hallmark of chronic fatigue syndrome/myalgic encephalomyelitis (CFS). This study examined brain structure variations associated with sleep quality in patients with CFS. 38 patients with CFS $(34.8 \pm 10.1$ years old) and 14 normal controls (NCs) $(34.7 \pm 8.4$ years old) were recruited. All subjects completed the Hospital Anxiety and Depression Scale (HADS), Pittsburgh sleep quality index (PSQI), and the Chalder fatigue scale (CFQ) questionnaires. Brain magnetic resonance imaging (MRI) measures included global and regional grey matter (GM) and white matter (WM) volumes, magnetization transfer T1 weighted (MT-T1w) intensities, and T1 weighted (T1w) and T2 weighted (T2w) spin-echo signal intensities. We performed voxel based group comparisons of these regional brain MRI measures and regressions of these measures with the PSQI and CFQ scales adjusted for age, anxiety and depression, and the appropriate global measure. In CFS patients, negative correlations were observed in the medial prefrontal cortex between PSQI and MTT1w intensities (family wise error corrected cluster, $\mathrm{P}_{\mathrm{FWE}}<0.05$ ) and between PSQI and T1w intensities $\left(\mathrm{P}_{\mathrm{FWE}}<0.05\right)$. In the same medial prefrontal cortex location, both MT and $\mathrm{T} 1 \mathrm{w}$ intensities were lower in CFS patients compared with NCs (uncorrected voxel P < 0.001). This study is the first to report that brain structural differences are associated with unrefreshing sleep in CFS. This result refutes the suggestion that unrefreshing sleep is a misperception in CFS patients and further investigation of this symptom is warranted.

Keywords: sleep quality, MRI, voxel based morphometry, medial prefrontal cortex, inferior fronto-occipital fasciculus.

Abbreviations: A\&D, anxiety and depression; $\mathrm{CFQ}$, Chalder fatigue scale; CFS, chronic fatigue syndrome; DARTEL, diffeomorphic anatomical registration through exponentiated lie algebra; FWE, family wise error; GM, grey matter; HADS, Hospital Anxiety and Depression Scale; IFOF, inferior fronto-occipital fasciculus; ME, myalgic encephalomyelitis; MNI, 
Montreal Neurological Institute; mPFC, medical prefrontal cortex; MRI, magnetic resonance imaging; MT-T1w, magnetization transfer T1 weighted; NC, normal control; PSG, polysomnography; PSQI, Pittsburgh sleep quality index; REM, rapid eye movement; SPM, statistical parametric mapping; T1w, T1 weighted; T2w, T2 weighted; VBIS, voxel-based iterative sensitivity; WM, white matter; 


\section{Introduction}

Chronic fatigue syndrome (CFS)/myalgic encephalomyelitis (ME) is a medically unexplained debilitating illness characterized by prolonged, post-exertional fatigue together with a range of other core symptoms ${ }^{1,2}$. There is ongoing debate as to whether CFS is the expression of somatic complaints accompanying a psychiatric syndrome, or a consequence of a viral infection, hormonal dysregulation, auto-immune disease and their interactions with the central nervous system and the autonomic nervous system ${ }^{3}$.

Sleep plays an important role in human health and well-being with its recuperative, restorative, and learning consolidation properties ${ }^{4,5}$. Unrefreshing sleep is a hallmark of ME/CFS that causes patients to wake up feeling tired even after extended periods of sleep, to experience excessive daytime sleepiness and to have difficulty falling asleep and staying asleep. Therefore, unrefreshing sleep results at least in worsening fatigue symptoms and adds to the 'illness burden' in CFS patients ${ }^{6}$. Roughly $91 \%$ of CFS patients report unrefreshing sleep regardless of adequate sleep duration ${ }^{7}$. The effect of unrefreshing sleep is of importance in CFS aetiology because sleep disturbance can cause fatigue, myalgia, and poor attention ${ }^{8}$. A recent path analysis study, exploring the interrelationship of different symptoms in CFS, found that poor sleep quality directly impacted on both mental and physical quality of life ${ }^{9}$. Similarly, another study found that subjective- but not actigraphy-defined sleep quality predicts next-day fatigue in patients with CFS ${ }^{10}$.

Despite consistent complaints of unrefreshing sleep by CFS patients, studies of sleep quality in CFS patients are limited. A review of 24 papers on sleep in CFS noted a discrepancy between objectively measured sleep architecture using polysomnography (PSG) and the multiple sleep latency test, and the subjective sleep quality reported by CFS patients ${ }^{11}$. Differences in PSG measures of total sleep time between CFS patients and healthy controls were limited. A twin study of 22 pairs of monozygotic twins where 1 member of the pair had 
CFS and the other did not, found that the twins did not differ in objective PSG measures of sleep latency, total sleep time, sleep efficiency, arousal number, arousal index, hypnogram awakenings, rapid eye movement (REM)-sleep latency, or percentages of stages 1, 2, and 3-4 (delta) sleep ${ }^{12}$. However, the percentage of REM sleep was increased in the CFS twins compared with the healthy twins. Other studies suggested that sleep homeostasis, reflected by the slow wave electroencephalogram, was impaired in CFS ${ }^{13,14}$.

Associations between sleep disturbance and brain structure changes have been observed in other diseases and aging. Mander et al. found that decreased sleep slow wave activity with age was mediated by a reduction in medical prefrontal cortex (mPFC) grey matter ${ }^{15}$. Similarly, sleep disturbance is associated with distinct alterations in default mode network functional connectivity in brain regions underpinning salient memory and sleep systems ${ }^{16}$. More specifically, sleep deprivation caused widespread changes in white matter microstructure ${ }^{17}$. Therefore, previous studies have shown that sleep disturbance was associated with brain abnormalities ${ }^{15-17}$. Given that unrefreshing sleep is a hallmark of CFS, we hypothesized that brain changes would be associated with unrefreshing sleep in patients with CFS. Interrogation of brain structure associations with sleep disturbance can provide direct in vivo evidence on whether there is brain involvement in unrefreshing sleep in CFS. To the best of our knowledge, there is no study of brain structure correlations with sleep quality in patients with CFS. Therefore, this study investigates if there are brain structural changes associated with unrefreshing sleep in CFS using magnetic resonance imaging (MRI). In addition to global and regional grey matter (GM) and white matter (WM) volumes, we performed a voxel-based quantitative analysis of magnetization transfer T1 weighted (MTT1w), T1 weighted (T1w), and T2 weighted (T2w) spin echo signal intensities. T1w hypointensities are associated histopathologically with severe tissue destruction, including axonal loss ${ }^{18}$. Similarly it has been shown that MT-T1w intensity is sensitive in depicting 
tissue lesions ${ }^{19}$. MT-T1w and T1w spin echo imaging are regarded as qualitative modalities because their intensities may be affected by factors such as shim, coil loading and receiver gain settings, and can vary markedly across imaging sessions and subjects. Therefore, we adopted the voxel-based iterative sensitivity (VBIS) method ${ }^{20}$, an objective statistical method for evaluation of signal intensity in our routinely acquired MT-T1w and T1w spin-echo images. The VBIS method has been validated against relaxation time imaging to effectively perform inter-group comparisons of MR spin echo intensities ${ }^{20}$. After signal normalization, $\mathrm{T} 1 \mathrm{w}$ and $\mathrm{T} 2 \mathrm{w}$ images are ideal for cross-sectional studies because of their low noise, high resolution and minimal distortion from patient-induced and instrumentation-induced magnetic field inhomogeneities ${ }^{21}$.

\section{Materials and Methods}

The study protocol was approved by the Human Research Ethics Committee of The Queen Elizabeth Hospital in compliance with the National Statement on Ethical Conduct in Human Research, and all subjects gave informed written consent for examinations.

\subsection{Subjects}

Thirty-eight CFS subjects from community-based specialist and general practice, meeting Canadian Consensus criteria ${ }^{22}$ for CFS, were assessed. Fourteen healthy, normal controls (NCs), unrelated to the CFS subjects, were recruited by public advertisement. The CFS group included 27 females and 11 males aged $34.8 \pm 10.1$ (mean \pm SD) years. The NC group included 10 females and 4 males aged $34.7 \pm 8.4$ years old. There was no significant difference in age $(\mathrm{P}=0.26)$ between the CFS and NC groups. No CFS or NC subject had developed a significant medical condition, including hypertension, or psychiatric illness during the research period. No subject was taking centrally acting medication. All subjects were right-handed, not smokers, nor substance abusers ascertained during clinician (RK) interview. 


\subsection{Symptom scores}

CFS severity was measured by the Chalder fatigue scale (CFQ), which discriminated reliably between the clinical and nonclinical conditions in 361 patients with CFS and 1615 NCs ${ }^{23}$. Higher CFQ scores indicate more severe disease. To determine their levels of depression and anxiety, all subjects completed the reliable and simple Hospital Anxiety and Depression Scale (HADS) questionnaire ${ }^{24}$. Sleep quality was evaluated using the Pittsburgh sleep quality index (PSQI), which has a sensitivity of $89.6 \%$ and specificity of $86.5 \%$ in distinguishing good and poor sleepers ${ }^{25}$. The PSQI is inversely proportional to the sleep quality, i.e. higher scores indicate poorer sleep quality.

\subsection{MRI acquisition}

MR images were acquired on a Philips 1.5T Intera MR scanner (Philips, Eindhoven, the Netherlands) with a body transmit coil and birdcage receive coil. Transverse anatomic images were acquired using a three-dimensional spoiled gradient-echo sequence with $\mathrm{TR}=5.76 \mathrm{~ms}$, $\mathrm{TE}=1.9 \mathrm{~ms}$, flip angle $=9^{\circ}$, resolution $=0.938 \times 0.938 \times 1 \mathrm{~mm}^{3}$. Transverse $\mathrm{T} 1 \mathrm{w} \mathrm{MR}$ images with $3 \mathrm{~mm}$ thick contiguous slices of $0.82 \times 0.82 \mathrm{~mm}^{2}$ pixels were acquired with TR $=600 \mathrm{~ms}, \mathrm{TE}=15 \mathrm{~ms}$, flip angle $=90^{\circ} . \mathrm{MT}-\mathrm{T} 1 \mathrm{w}$ MR images were acquired with offresonance excitation followed by $\mathrm{T} 1$ fast spin echo read-out for $3 \mathrm{~mm}$ thick contiguous slices of $1.19 \times 1.19 \mathrm{~mm}^{2}$ pixels and $\mathrm{TR}=600 \mathrm{~ms}, \mathrm{TE}=15 \mathrm{~ms}$, flip angle $=90^{\circ}$. Transverse T2w MR image with $3 \mathrm{~mm}$ thick contiguous slices of $0.82 \times 0.82 \mathrm{~mm}^{2}$ pixels were acquired with $\mathrm{TR}=4000 \mathrm{~ms}, \mathrm{TE}=80 \mathrm{~ms}$, flip angle $=90^{\circ}$.

\subsection{MRI processing}

The three-dimensional anatomic images were segmented into GM, WM, and cerebrospinal fluid (CSF) images using SPM12 (Wellcome Trust Centre for Neuroimaging, London, UK), in which a unified probabilistic framework that combined image segmentation, tissue classification, and bias correction within the same generative model was implemented ${ }^{26}$. The 
empirical parameter for Markov Random Field removal of isolated voxel assignments, was set to 4 , in accordance with our previous validation results ${ }^{27}$. Global GM and WM volumes were computed in SPM12 by summing all voxel values in the GM and WM images.

Voxel based morphometry (VBM) in SPM12 was used to evaluate regional volume changes in the brain. VBM is a well-established tool to examine patterns of regional anatomical brain changes in neurological diseases and neuroanatomical correlates of CNS disease symptoms. VBM spatially normalizes GM and WM images for each subject from their native space to MNI space and the local GM and WM volume changes associated with their non-linear deformations are incorporated into their GM and WM image intensities ${ }^{28}$. More specifically, a study specific template was constructed from the GM images of all subjects normalized using DARTEL (diffeomorphic anatomical registration through exponentiated lie algebra) implemented in SPM12 ${ }^{29}$. Deformations to the new template were refined over 6 iterations. The study specific template was then normalized to a standard anatomical space defined by the Montreal Neurological Institute (MNI) atlas. The DARTEL flow field images representing individual image deformations to the final study-specific template, and the transformation of the template to MNI space, were combined. Then the regional GM and WM volumetric changes associated with the local composite deformation were encoded onto their intensities to form 'modulated' images, followed by a Gaussian smoothing with an $8 \mathrm{~mm}$ $\times 8 \mathrm{~mm} \times 8 \mathrm{~mm}$ kernel.

The T1w, MT-T1w, T2w images were firstly co-registered to their corresponding anatomic images. Secondly, co-registered T1w, MT, and T2w images were normalized to MNI space using the combined deformations computed above and smoothed with an $8 \mathrm{~mm} \times 8 \mathrm{~mm} \times$ 8mm Gaussian kernel. Global signal levels for each individual and each modality were computed for each subject as the mean voxel value in a mask (brain region) generated using 
the VBIS method ${ }^{20}$. The MT-T1w, T1w, and T2w signal intensities were normalized using their corresponding global signal levels.

\subsection{Statistical analysis of symptom scores and global volumes}

The following statistical tests of symptom scores and global GM and WM volumes were performed using SPSS22 (IBM, New York). An independent two sample two tailed t-test was used to compare inter-group differences in clinical measures (CFQ, anxiety and depression from HADS, and PSQI), global GM volumes, and global WM volumes. The Bonferroni correction was used to counteract multiple comparisons of 6 characteristics $(\mathrm{P}<0.0083)$. Pearson correlations between each clinical measure and global GM and WM volumes from all subjects and separately from patients with CFS were calculated. Bivariate tests of significant difference in correlation coefficient from zero with two tails were followed with Bonferroni correction for multiple comparisons of 15 pairs to test for uncorrected $(\mathrm{P}<0.05)$ and corrected $(\mathrm{P}<0.003)$ statistical significance.

\subsection{Voxel-based statistical analysis of MRI}

Voxel based statistical analysis of the processed MRI was performed with SPM12. Cluster statistical inference was used for the primary detection of group differences and correlations with clinical variables.

A two sample t-test was used to compare the CFS and NC groups for regional GM and WM volumetric differences (modulated GM images and WM images) and regional differences in signal intensity for T1w, MT-T1w, and T2w. All comparisons incorporated age as a nuisance covariate. The significance of inter-group differences was tested using family wise error (FWE) corrected cluster $\mathrm{P}$ value $\left(\mathrm{P}_{F W E_{-} \text {corr_cluster }}\right)<0.05$ with a cluster forming voxel threshold of uncorrected $\mathrm{P}<0.001\left(\mathrm{P}_{\text {uncorr_voxel }}<0.001\right)$.

Voxel-wise statistical parametric mapping (SPM) regressions were performed between images from the CFS group against the symptom scores of CFQ and PSQI. All regressions 
incorporated the covariates of age, the appropriate global value, and HADS anxiety and depression (A\&D) scores. The significance of correlation was tested using $\mathrm{P}_{F W E \_c o r r}$ cluster $<$ 0.05 with cluster forming threshold of $\mathrm{P}_{\text {uncorr_voxel }}<0.001$.

When a significant cluster was detected in either a group level difference or a correlation in CFS group analysis, a secondary statistical assessment was conducted at its location looking at voxel statistics in order to test whether the correlation also existed in all subjects (CFS $+\mathrm{NC}$ ) and in the NC group. Thus, detection of a significant cluster in a group test was followed by examination of voxel $\mathrm{P}$ from the correlation tests at the same location in that image type, and vice versa. Because of the a-priori nature of locations assessed in this second step, uncorrected voxel $\mathrm{P}$ were used and the SPM voxel significance threshold was $\mathrm{P}_{\text {uncorr_voxel }}$ $<0.001$.

For the regions with significant correlations with symptom scores in the CFS group, voxelwise SPM regressions were performed for all subjects $(\mathrm{CFS}+\mathrm{NC})$ versus the same symptom scores. Similar regressions were also performed using images from NCs only. Further, voxel based regressions with symptom scores in images from CFS were performed without adjustment for anxiety and depression in HADS. For these regions, the significance of correlation were tested using $\mathrm{P}_{\text {uncorr_voxel }}<0.001$. The inter-group comparisons were also revisited with relaxed significant threshold of $\mathrm{P}_{\text {uncorr_voxel }}<0.001$ in these regions.

\section{Results}

\subsection{Symptom score and global GM and WM volumes}

The mean values and the significances of differences between CFS and NC groups for symptom scores and global GM and WM volumes are summarized in Table 1. There was no significant difference in total GM and WM volumes between CFS and NC. All symptom scores were significantly different between CFS and NC. Bivariate correlations between any pair of symptom scores or total GM and WM volumes in CFS patients only and in all 
subjects $(\mathrm{CFS}+\mathrm{NC})$ are summarized in Table 2. In the CFS group, significant correlations were detected between total GM \& WM volumes and between depression \& anxiety. In addition, CFQ \& PSQI, CFQ \& anxiety, and CFQ \& depression, PSQI \& anxiety were correlated with uncorrected $\mathrm{P}<0.05$. Except for the correlation between total GM and WM volumes, these correlations were stronger when the CFS and NC groups were pooled.

\subsection{Voxel-wise SPM of inter-group differences}

Regional WM volumes in the left inferior fronto-occipital fasciculus (IFOF) were significantly lower $\left(\mathrm{P}_{F W E_{-} \text {corr_cluster }}=0.005\right)$ in patients with CFS than those in NCs (Fig 1). The SPM statistics for this regional difference were as follows: cluster size 291 voxels, peak $\mathrm{T}$ score 5.73, peak MNI coordinates [-52 -12 -16]. The secondary statistical assessment showed an adjacent negative correlation between regional WM volumes and PSQI $\left(\mathrm{P}_{\text {uncorr_voxel }}<0.001\right)$ : cluster size 24 voxels, peak T score 3.73, peak MNI coordinates [-56 $15-22]$ in the CFS group. There were no significant differences $\left(\mathrm{P}_{F W E_{-} \text {corr_cluster }}>0.05\right)$ in regional GM volume, MT-T1w, T1w or T2w between the CFS and NC groups.

\subsection{Voxel-wise PSQI regressions}

The MT-T1w and T1w intensities were significantly and negatively correlated with PSQI $\left(\mathrm{P}_{F W E_{c} \text { corr_cluster }}<0.05\right)$ in multiple regions, including the medial prefrontal cortex $(\mathrm{mPFC})$, internal capsule, and right IFOF (Figs 2 and 3). The secondary statistical assessment of intergroup differences showed lower MT-T1w and T1w intensities $\left(\mathrm{P}_{\text {uncorr_voxel }}<0.001\right)$ in the $\mathrm{mPFC}$ in CFS. The relative locations of all $\mathrm{mPFC}$ results are shown in Fig 4. There was no inter-group difference $\left(\mathrm{P}_{\text {uncorr_voxel }}>0.001\right)$ of MT-T1w or T1w intensity in the right internal capsule or right IFOF. We did not observe significant PSQI correlations $\left(\mathrm{P}_{F W E}\right.$ corr_cluster $>$ 0.05 ) for regional GM volumes, WM volumes, or T2W intensities.

\subsection{Voxel-wise CFQ regressions}


$\mathrm{T} 1 \mathrm{w}$ intensities in the right insula were significantly and negatively correlated $\left(\mathrm{P}_{F W E}\right.$ corr_cluster $=0.002)$ with CFQ in CFS: cluster size 534 voxels, peak T score 6.38, peak MNI coordinates [46 -8 14] (Fig 5). When all subjects were included, the peak T score became lower but with increased cluster size: $\mathrm{P}_{F W E_{-} \text {corr_cluster }}=1.4 \mathrm{e}-5$, cluster size 1263 voxels, peak $\mathrm{T}$ score 5.49, peak MNI coordinate [45 -8 9]. There was no significant correlation $\left(\mathrm{P}_{\text {uncorr_voxel }}>0.001\right)$ between T1w intensities and CFQ in the right insula in images from NCs only. No significant inter-group difference of $\mathrm{T} 1 \mathrm{w}$ intensities between $\mathrm{CFS}$ and $\mathrm{NC}$ groups $\left(\mathrm{P}_{\text {uncorr_voxel }}>0.001\right)$ in the right insula was observed and we did not observe any significant CFQ correlations with regional GM volumes, WM volumes, MT-T1w intensities, or T2w intensities in the CFS group.

\section{Discussion}

This is the first brain MRI study in CFS to test for brain structure associations with the frequent complaint of unrefreshing sleep as measured by the PSQI. There were three novel findings. 1) In the mPFC, MT-T1w and T1w intensities are significantly correlated with the sleep quality measure PSQI. Furthermore, the mPFC MT-T1w and T1w intensities were lower in patients with CFS than those from NCs. 2) The regional WM volumes in the left IFOF are significantly lower in patients with CFS than in NCs. In CFS subjects WM volumes in the left IFOF correlated with PSQI. 3) The MT-T1w and T1w intensities in the right internal capsule were significantly correlated with PSQI both in the CFS group alone and in the combined CFS+NC groups, but not in the NC group alone. The $\mathrm{T} 1 \mathrm{w}$ intensities in the right insula were significantly correlated with the CFS severity measure CFQ both in the CFS group and in the combined group. MT-T1w intensities in the right IFOF were also significantly correlated with PSQI in both the CFS and combined groups. However, no intergroup difference in MT-T1w or T1w level measures was observed in the corresponding regions. 
This study found that MT-T1w and T1w intensities were negatively correlated with PSQI in the mPFC, i.e. impaired sleep quality is associated with lower MT-T1w and T1w intensities. MT-T1w hypointensities indicate a reduced level of macromolecules in brain tissue or a reduced capacity for them to exchange magnetization with the surrounding water molecules. In white matter this would most likely indicate changes in myelin. However, the mPFC locations involved here were in grey matter where MT-T1w hypointensity has been interpreted to indicate tissue matrix damage and axon loss ${ }^{30}$. A post-mortem study in Multiple Sclerosis confirmed that T1w hypointensities were histologically correlated with tissue matrix damage and axon loss ${ }^{18}$. Therefore, our findings suggest that unrefreshing sleep is associated with mPFC matrix damage and axon loss. Our mPFC finding is consistent with previous findings that mPFC atrophy diminishes slow wave activity during non-rapid eye movement sleep ${ }^{15}$. Experimental support for the role of mPFC in mediating sleep has been reviewed ${ }^{31}$. The correlations between mPFC MT-T1w intensities and PSQI were stronger with adjustment for anxiety and depression (Fig.2). Similarly, the correlations between T1w intensities and PSQI became more significant with adjustment for anxiety and depression (Fig. 3). Thus, while anxiety and depression contribute variance to these MRI measures in the mPFC, this variance is not correlated with the variance associated with PSQI. Removal of anxiety and depression variance therefore strengthens the statistics of the MRI versus PSQI signal. Although PSQI correlates with depression and anxiety at the subject level, their correlations with local MRI measures are different.

Unrefreshing sleep is a defining characteristic of CFS. However, lack of objective evidence has led to questioning of subjective patient reports ${ }^{12,32}$. This study not only established negative correlations of MT-T1w with PSQI and T1w with PSQI in the mPFC, but also found lower mPFC MT-T1w and T1w intensities in CFS patients than those in NCs. These two findings together confirmed that changes in MRI characteristics in the mPFC were associated 
with unrefreshing sleep in CFS patients, although causality between them could not be determined. It is not necessary, therefore, to resort to subject misperceptions to explain unrefreshing sleep in CFS. Our findings suggest traditional sleep measures may not be able to detect sleep abnormality in CFS patients. The reasons for the changed MRI characteristics in the mPFC in CFS could not be determined in this study, although it is noteworthy that mPFC reduction of serotonin transporters and $\mathrm{mPFC}$ hypoperfusion were observed in previous studies of $\mathrm{CFS}^{33,34}$.

Regional IFOF WM volumes were significantly decreased here in patients with CFS compared with NCs. This finding supports our previous observation of progressive WM atrophy in the left IFOF in CFS ${ }^{27}$ although 15 of the 40 CFS patients and 10 of the 14 NCs in this study were also used in that longitudinal study. WM atrophy in the left IFOF also correlated with PSQI. To the best of our knowledge, the left IFOF does not play a role in mediating sleep, although a recent study found frontoparietal connection strength predicts individual resistance to sleep deprivation ${ }^{35}$. Therefore, left IFOF WM atrophy could exacerbate poor sleep quality in patients with CFS.

We observed significant and negative correlations of MT-T1w \& PSQI and T1w \& PSQI in right internal capsule WM in CFS patients. The statistical assessments suggested that this relationship does not involve anxiety and depression and also exists in NCs. The internal capsule mainly contains the corticospinal tract which carries sensorimotor information between the motor cortex neurons and motor neurons in the spinal cord that control limb muscles. This observation may explain the clinical presentation of difficulties in spatial sensing and coordination of motor function in CFS although we are not able to make decisive conclusions given that no significant group difference was observed. The internal capsule also connects the thalamus with the insular cortex, comprising the interoceptive pathway for body state awareness and pain experience ${ }^{36}$. Therefore, these relationships consolidate the 
negative correlation of $\mathrm{T} 1 \mathrm{w} \& \mathrm{CFQ}$ in the right insula cortex. These findings are also consistent with a recent report that an aberration of the salience network might play a role in CFS pathophysiology ${ }^{37}$. However, we did not observe inter-group differences in these regions. This could be due to the small sample size of NCs or indicate that these relationships exist in all individuals and are not specifically related to CFS. Similarly, we were not able to make a CFS-related conclusion on correlations between MT-T1w \& PSQI in the right IFOF because inter group differences were not observed.

This study was limited in several aspects due to its retrospective nature. The MT-T1w, T1w, and $\mathrm{T} 2 \mathrm{w}$ spin echo imaging are qualitative modalities because their intensities may be affected by factors such as shim, coil loading and receiver gain settings, and can vary markedly across imaging sessions and subjects. Therefore, we adopted the VBIS analysis method which had been validated against relaxation time imaging as effective in cross group comparison of MR spin echo intensities ${ }^{20}$. Although VBIS cannot correct for regional intensity differences caused by heterogeneity in receiving coil sensitivity, we argue that these differences would introduce additional variance and decrease statistical sensitivity, but would not introduce bias between groups. The sample size of the NC group is small in this study due to the retrospective nature of this analysis. Similarly, VBM can be sensitive to artifacts including normalization errors and misclassification of tissue types. However, all these may confound the statistical analysis and decrease the sensitivity, but are less likely to increase the chance of false positives given that MRI data of CFS and NCs were collected and processed in an indistinguishable way. Furthermore, the overlapping of clusters in both MT-T1w and T1w regression and group difference analysis suggested a low probability of false positive findings. Therefore, although we are able to confirm mPFC as one brain structural underpinning of disrupted sleep in CFS, we cannot rule out that other structural changes may 
also be involved, especially in regions having correlations with PSQI that here show no intergroup difference.

\section{Conclusion}

This is the first study to investigate brain structure associations with unrefreshing sleep in CFS. We found that mPFC MRI measures correlate with sleep quality and concluded that mPFC integrity may be affected in patients with CFS. The discrepancy between subjective sleep quality reports in CFS and the objective instrumental measures most likely arises because traditional sleep monitoring methods do not respond to the mechanism that causes unrefreshing sleep in CFS. This study warrants the exploration in more depth of unrefreshing sleep in CFS patients.

\section{Acknowledgments}

Important imaging support was provided by The Queen Elizabeth Hospital Radiology Department. 


\section{References}

1. Carruthers BM, Jain AK, De Meirleir KL, Peterson DL, Klimas NG, Lerner AM, Bested AC, Flor-Henry P, Joshi P, Powles AP. Myalgic encephalomyelitis/chronic fatigue syndrome: clinical working case definition, diagnostic and treatment protocols. J Chronic Fatigue Syndr. 2003;11(1):7-115.

2. Fukuda K, Straus SE, Hickie I, Sharpe MC, Dobbins JG, Komaroff A. The chronic fatigue syndrome: a comprehensive approach to its definition and study. Ann Intern Med. 1994;121:953-959.

3. Holgate S, Komaroff A, Mangan D, Wessely S. Chronic fatigue syndrome: understanding a complex illness. Nat Rev Neurosci. 2011;12:539-544.

4. Van Cauter E, Leproult R, Plat L. Age-related changes in slow wave sleep and REM sleep and relationship with growth hormone and cortisol levels in healthy men. JAMA. 2000;284(7):861-868.

5. Walker MP, Stickgold R. Sleep-dependent learning and memory consolidation. Neuron. 2004;44(1):121-133.

6. Gotts ZM, Newton JL, Ellis JG, Deary V. The experience of sleep in chronic fatigue syndrome: A qualitative interview study with patients. $\mathrm{Br} J$ Health Psychol. 2016;21(1):71-92.

7. Nisenbaum R, Jones JF, Unger ER, Reyes M, Reeves WC. A population-based study of the clinical course of chronic fatigue syndrome. Health Qual Life Outcomes. 2003;1:49.

8. Morriss RK, Wearden AJ, Battersby L. The relation of sleep difficulties to fatigue, mood and disability in chronic fatigue syndrome. J Psychosom Res. 1997;42(6):597605.

9. Tobback E, Hanoulle I, Mariman A, Delesie L, Pevernagie D, Vogelaers D. Factors determining fatigue in the chronic fatigue syndrome: a path analysis. Acta Clinica Belgica. 2016:1-6.

10. Russell C, Wearden AJ, Fairclough G, Emsley RA, Kyle SD. Subjective but Not Actigraphy-Defined Sleep Predicts Next-Day Fatigue in Chronic Fatigue Syndrome: A Prospective Daily Diary Study. Sleep. 2016;39(4):937-944.

11. Jackson ML, Bruck D. Sleep abnormalities in chronic fatigue syndrome/myalgic encephalomyelitis: a review. Journal of clinical sleep medicine : JCSM : official publication of the American Academy of Sleep Medicine. 2012;8(6):719-728.

12. Watson NF, Kapur V, Arguelles LM, Goldberg J, Schmidt DF, Armitage R, Buchwald D. Comparison of subjective and objective measures of insomnia in monozygotic twins discordant for chronic fatigue syndrome. Sleep. 2003;26(3):324328.

13. Decker MJ, Tabassum H, Lin JM, Reeves WC. Electroencephalographic correlates of Chronic Fatigue Syndrome. Behav Brain Funct. 2009;5:43.

14. Armitage R, Landis C, Hoffmann R, Lentz M, Watson NF, Goldberg J, Buchwald D. The impact of a 4-hour sleep delay on slow wave activity in twins discordant for chronic fatigue syndrome. Sleep. 2007;30(5):657-662.

15. Mander BA, Rao V, Lu B, Saletin JM, Lindquist JR, Ancoli-Israel S, Jagust W, Walker MP. Prefrontal atrophy, disrupted NREM slow waves and impaired hippocampal-dependent memory in aging. Nat Neurosci. 2013;16(3):357-364.

16. McKinnon AC, Lagopoulos J, Terpening Z, Grunstein R, Hickie IB, Batchelor J, Lewis SJ, Duffy S, Shine JM, Naismith SL. Sleep disturbance in mild cognitive impairment is associated with alterations in the brain's default mode network. Behav Neurosci. 2016;130(3):305-315. 
17. Elvsashagen T, Norbom LB, Pedersen PO, Quraishi SH, Bjornerud A, Malt UF, Groote IR, Westlye LT. Widespread changes in white matter microstructure after a day of waking and sleep deprivation. PLoS One. 2015;10(5):e0127351.

18. van Walderveen MA, Kamphorst W, Scheltens P, van Waesberghe JH, Ravid R, Valk J, Polman CH, Barkhof F. Histopathologic correlate of hypointense lesions on T1weighted spin-echo MRI in multiple sclerosis. Neurology. 1998;50(5):1282-1288.

19. da Rocha AJ, Oliveira AS, Fonseca RB, Maia AC, Jr., Buainain RP, Lederman HM. Detection of corticospinal tract compromise in amyotrophic lateral sclerosis with brain MR imaging: relevance of the T1-weighted spin-echo magnetization transfer contrast sequence. AJNR Am J Neuroradiol. 2004;25(9):1509-1515.

20. Abbott D, Pell G, Pardoe H, Jackson G. Voxel-Based Iterative Sensitivity (VBIS): methods and a validation of intensity scaling for T2-weighted imaging of hippocampal sclerosis. NeuroImage. 2009;44:812-819.

21. Bushberg J, Seibert J, Leidholdt E, Boone J. The essential physics of medical imaging. 2nd ed. Philadelphia: Lippincott, Williams and Wilkins; 2002.

22. Carruthers B, van de Sande M, DeMeirleir K, Klimas N, Broderick G, Mitchell T, Staines D, Powles A, Speight N, Vallings R, Bateman L, Baumgarten-Austrheim B, Bell D, Carlo-Stella N, Chia J, Darragh A, Lewis D, Jo D, Light A, MarshallGradisnik S, Mena I, Mikovits J, Miwa K, Murovska M, Pall M, Stevens S. Myalgic encephalomyelitis: International Consensus Criteria. J Intern Med. 2011;270:327 338.

23. Cella M, Chalder T. Measuring fatigue in clinical and community settings. $J$ Psychosom Res. 2010;69(1):17-22.

24. Snaith RP. The Hospital Anxiety And Depression Scale. Health Qual Life Outcomes. 2003;1:29.

25. Buysse DJ, Reynolds CF, 3rd, Monk TH, Berman SR, Kupfer DJ. The Pittsburgh Sleep Quality Index: a new instrument for psychiatric practice and research. Psychiatry Res. 1989;28(2):193-213.

26. Ashburner J, Friston KJ. Unified segmentation. NeuroImage. 2005;26(3):839-851.

27. Shan ZY, Kwiatek R, Burnet R, Del Fante P, Staines DR, Marshall-Gradisnik SM, Barnden LR. Progressive brain changes in patients with chronic fatigue syndrome: A longitudinal MRI study. J Magn Reson Imaging. 2016;44(5):1301-1311.

28. Ashburner J, Friston KJ. Voxel-based morphometry--the methods. NeuroImage. 2000;11(6 Pt 1):805-821.

29. Ashburner J. A fast diffeomorphic image registration algorithm. NeuroImage. 2007;38:95-113.

30. Wolff SD, Balaban RS. Magnetization transfer contrast (MTC) and tissue water proton relaxation in vivo. Magn Reson Med. 1989;10(1):135-144.

31. Muzur A, Pace-Schott EF, Hobson JA. The prefrontal cortex in sleep. Trends Cogn Sci. 2002;6(11):475-481.

32. Reeves WC, Heim C, Maloney EM, Youngblood LS, Unger ER, Decker MJ, Jones JF, Rye DB. Sleep characteristics of persons with chronic fatigue syndrome and nonfatigued controls: results from a population-based study. BMC Neurol. 2006;6:41.

33. Shungua D, Weiduschata N, Murrough J, Maoa X, Pillemer S, Dykea J, Medow M, Natelson B, Stewart J, Mathew S. Increased ventricular lactate in chronic fatigue syndrome. III. Relationships to cortical glutathione and clinical symptoms implicate oxidative stress in disorder pathophysiology. NMR Biomed. 2012;25:1073-1087.

34. Yamamoto S, Ouchi Y, Onoe H, Yoshikawa E, Tsukada H, Takahashi H, Iwase M, Yamaguti K, Kuratsune H, Watanabe Y. Reduction of serotonin transporters of patients with chronic fatigue syndrome. NeuroReport. 2004;17:2571-2574. 
35. Cui J, Tkachenko O, Gogel H, Kipman M, Preer LA, Weber M, Divatia SC, Demers LA, Olson EA, Buchholz JL, Bark JS, Rosso IM, Rauch SL, Killgore WD. Microstructure of frontoparietal connections predicts individual resistance to sleep deprivation. NeuroImage. 2015;106:123-133.

36. Bushnell MC, Ceko M, Low LA. Cognitive and emotional control of pain and its disruption in chronic pain. Nat Rev Neurosci. 2013;14(7):502-511.

37. Wortinger LA, Endestad T, Melinder AM, Oie MG, Sevenius A, Bruun Wyller V. Aberrant Resting-State Functional Connectivity in the Salience Network of Adolescent Chronic Fatigue Syndrome. PLoS One. 2016;11(7):e0159351. 


\section{Figure Legends}

Fig 1. Results from voxel based comparison of regional white matter (WM) volumes between chronic fatigue syndrome (CFS) and normal control (NC) groups. The cluster $(\mathrm{CFS}<\mathrm{NC})$ is superimposed on sections through its peak voxel of the 3D T1 weighted Montreal Neurological Institute (MNI) brain atlas. Colour coding from yellow to red represents T statistic values from 3.0 to 7.0.

Fig 2. Results from voxel based regressions of the Pittsburgh sleep quality index (PSQI) with magnetization transfer T1 weighted (MT-T1w) spin echo intensities. The images show three significant clusters from the analysis of the chronic fatigue syndrome (CFS) group adjusted for anxiety and depression (A\&D). Clusters were located in the medial prefrontal cortex (A), the internal capsule (B) and the right IFOF (C). Each cluster is superimposed on sections through its peak voxel of the 3D T1 weighted Montreal Neurological Institute (MNI) brain atlas. Colour coding from yellow to red represents $\mathrm{T}$ statistic values from 3.0 to 7.0. The tables on the right summarise MT-T1w versus PSQI cluster properties for regression analysis of pooled subjects $(\mathrm{CFS}+\mathrm{NC}), \mathrm{CFS}$ only (with and without A\&D adjustment) and NC only. PFWE is the family wise error (FWE) corrected cluster P value (cluster forming voxel $\mathrm{P}<0.001) ; \mathrm{k}_{\mathrm{E}}$ is the cluster size in voxels; $\mathrm{T}$ is the T-statistic of the peak voxel; MNI is the peak voxel coordinates in MNI space; ae-b represents $\mathrm{a} \times 10^{-\mathrm{b}}$.

Fig 3. Results from voxel based regressions of the Pittsburgh sleep quality index (PSQI) with T1 weighted spin echo (T1w) intensities. The images on the left show two clusters where regional T1w intensities correlate with PSQI in the chronic fatigue syndrome (CFS) group after controlling for anxiety and depression (A\&D). Clusters were located in the medial prefrontal cortex (A) and the internal capsule (B). Each cluster is superimposed on sections through its peak voxel of the 3D T1 weighted Montreal Neurological Institute (MNI) brain atlas. Colour coding from yellow to red represents $\mathrm{T}$ statistic values from 3.0 to 7.0. Tables 
on the right summarise cluster properties from T1w versus PSQI for normal controls (NC) only, pooled subjects $(\mathrm{NC}+\mathrm{CFS}$ ), and from the CFS only (with and without A\&D adjustment). $\mathrm{P}_{\mathrm{FWE}}$ is the family wise error (FWE) corrected cluster $\mathrm{P}$ value (cluster forming voxel $\mathrm{P}<0.001) ; \mathrm{k}_{\mathrm{E}}$ is the cluster size in voxels; $\mathrm{T}$ is the T-statistic of the peak voxel; MNI is the peak voxel coordinates in MNI space.

Fig 4. Four clusters in the medial prefrontal cortex from magnetization transfer $\mathbf{T 1}$ weighted spin echo (MT-T1w) and conventional T1 weighted spin echo (T1w) intensity analyses. For both MT-T1w and T1w, clusters are shown for the group comparison (CFS vs NC) and the CFS regression with the Pittsburgh sleep quality index (PSQI). Significant correlations (family wise error corrected cluster $\mathrm{P}$ value, $\mathrm{P}_{\mathrm{FWE}}<0.05$ ) between MT-T1w intensities and the PSQI indices and between conventional T1w intensities and the PSQI indices (yellow and blue) are superimposed on the average T1 brain atlas in Montreal Neurological Institute (MNI) space. Two sample t-tests comparing MT-T1w and T1w intensities between CFS and normal control (NC) groups (green and red) also showed significant differences (uncorrected voxel level $\mathrm{P}<0.001$ ) in the same region.

Fig 5. Results from voxel based regressions of the Chalder fatigue scale (CFQ) with T1 weighted spin echo (T1w) intensities. The cluster shows the location in the right insula where regional $\mathrm{T} 1 \mathrm{w}$ intensities correlate with $\mathrm{CFQ}$ in the chronic fatigue syndrome (CFS) group adjusted for anxiety and depression (A\&D). The cluster is superimposed on sections through its peak voxel of the 3D T1 weighted Montreal Neurological Institute (MNI) brain atlas. Colour coding from yellow to red represents $\mathrm{T}$ statistic values from 3.0 to 7.0. 
Table 1. Means of symptom scores and global GM and WM volumes of CFS and NC

\begin{tabular}{llll}
\multicolumn{4}{c}{ groups $^{\S}$} \\
\hline Characteristics & $\mathrm{M}_{\mathrm{CFS}} \pm \mathrm{SD}_{\mathrm{CFS}}$ & $\mathrm{M}_{\mathrm{NC}} \pm \mathrm{SD}_{\mathrm{NC}}$ & $\mathrm{P}$ \\
\hline $\mathrm{GM}$ & $666.3 \pm 71.9 \mathrm{ml}$ & $697.2 \pm 94.2 \mathrm{ml}$ & 0.28 \\
$\mathrm{WM}$ & $504.4 \pm 66.4 \mathrm{ml}$ & $514.0 \pm 45.4 \mathrm{ml}$ & 0.55 \\
CFQ & $22.5 \pm 7.2$ & $3.5 \pm 3.3$ & $<0.008$ \\
PSQI & $9.7 \pm 4.0$ & $4.0 \pm 2.4$ & $<0.008$ \\
Anxiety & $7.1 \pm 4.2$ & $4.4 \pm 2.3$ & $<0.008$ \\
Depression & $6.1 \pm 3.5$ & $1.8 \pm 2.1$ & $<0.008$
\end{tabular}

§: GM: grey matter, WM: white matter, CFS: chronic fatigue syndrome, NC: normal control, M: mean value, SD: standard deviation P: significance level of differences between CFS and NC groups, CFQ: Chalder fatigue scale, PSQI: Pittsburgh sleep quality index. 
Table 2. Bivariate correlations among symptom scores and total GM and WM volumes ${ }^{\S}$

\begin{tabular}{lcccccc}
\hline Characteristics & GM & WM & CFQ & PSQI & Anxiety & Depression \\
\hline GM & & $\mathbf{0 . 6 4}^{\mathbf{a}}$ & -0.15 & 0.07 & -0.06 & 0.03 \\
WM & $\mathbf{0 . 6 7}^{\mathbf{a}}$ & & -0.10 & 0.12 & -0.02 & 0.14 \\
CFQ & -0.13 & -0.14 & & $\mathbf{0 . 6 0}^{\mathbf{a}}$ & $\mathbf{0 . 4 9}^{\mathbf{a}}$ & $\mathbf{0 . 6 3}^{\mathbf{a}}$ \\
PSQI & 0.23 & 0.31 & $\mathbf{0 . 3 1}^{\mathbf{b}}$ & & $\mathbf{0 . 4 5}^{\mathbf{a}}$ & $\mathbf{0 . 4 8}^{\mathbf{a}}$ \\
Anxiety & -0.05 & 0.01 & $\mathbf{0 . 4 4}^{\mathbf{b}}$ & $\mathbf{0 . 3 3}^{\mathbf{b}}$ & & $\mathbf{0 . 6 3}^{\mathbf{a}}$ \\
Depression & -0.06 & 0.16 & $\mathbf{0 . 4 1}^{\mathbf{b}}$ & 0.26 & $\mathbf{0 . 6 1}^{\mathbf{a}}$ & \\
\hline
\end{tabular}

$\S$ The bivariate correlation coefficients in the chronic fatigue syndrome (CFS) group only are listed to the left and below the diagonal. Coefficients for all subjects (CFS + NC) are listed in the shaded cells to the right and above the diagonal. CFQ: Chalder fatigue scale, PSQI: Pittsburgh sleep quality index, ${ }^{\mathrm{a}}$ correlation significance level $\mathrm{P}<0.002,{ }^{\mathrm{b}}$ correlation significance level $\mathrm{P}<0.05$. 


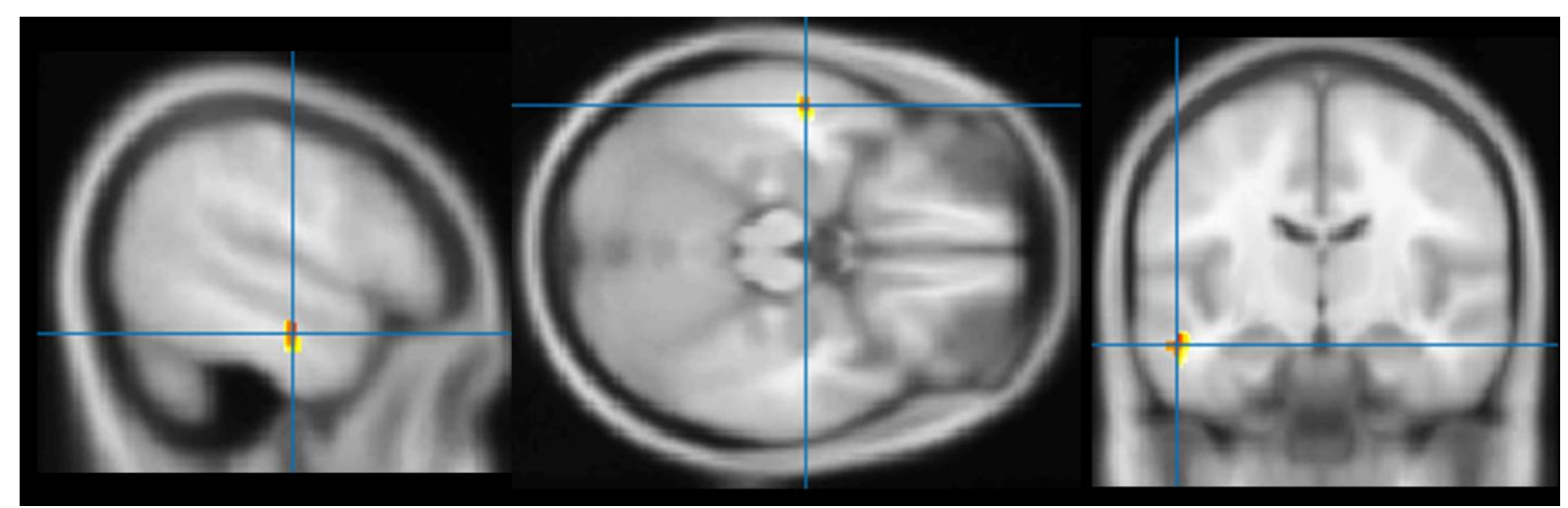

Fig 1. Results from voxel based comparison of regional white matter (WM) volumes between chronic fatigue syndrome (CFS) and normal control (NC) groups. 


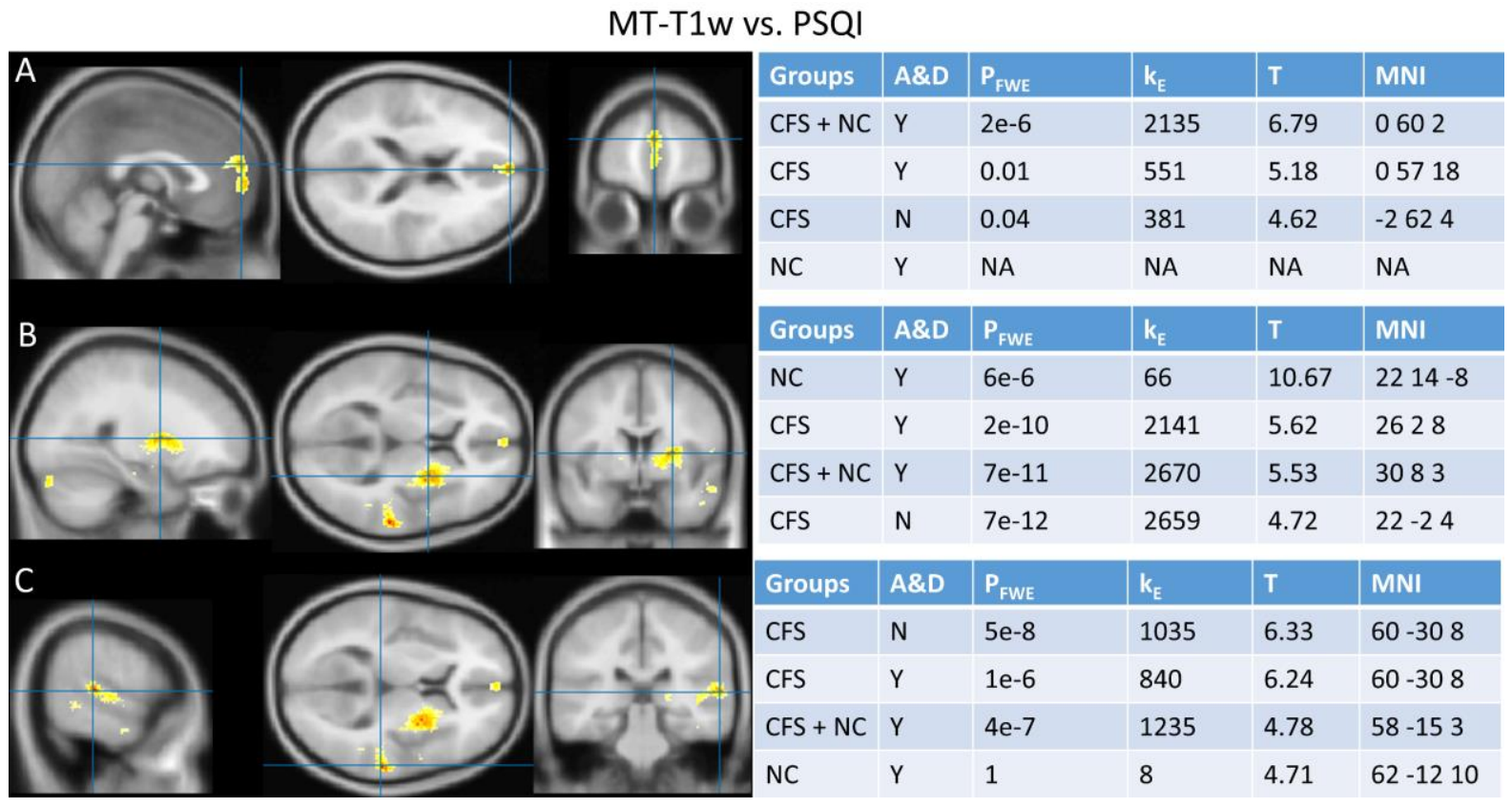

Fig 2. Results from voxel based regressions of the Pittsburgh sleep quality index (PSQI) with magnetization transfer T1 weighted (MT-T1w) spin echo intensities. 
T1w vs. PSQI

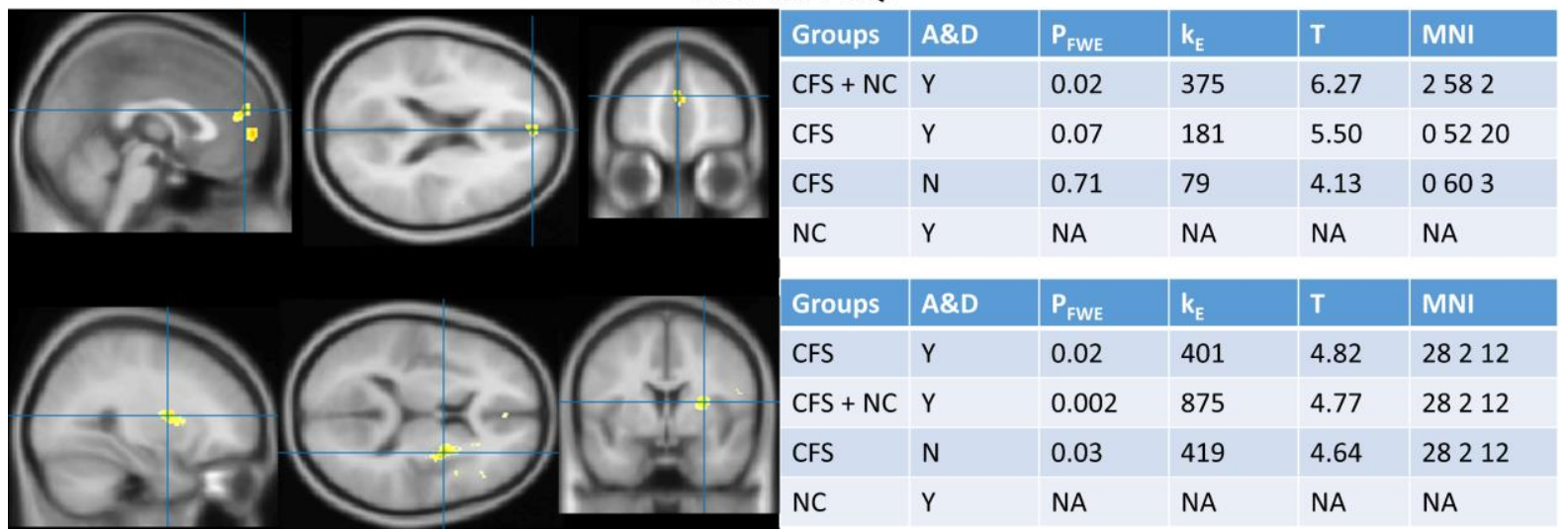

Fig 3. Results from voxel based regressions of the Pittsburgh sleep quality index (PSQI) with $\mathrm{T} 1$ weighted spin echo (T1w) intensities. 


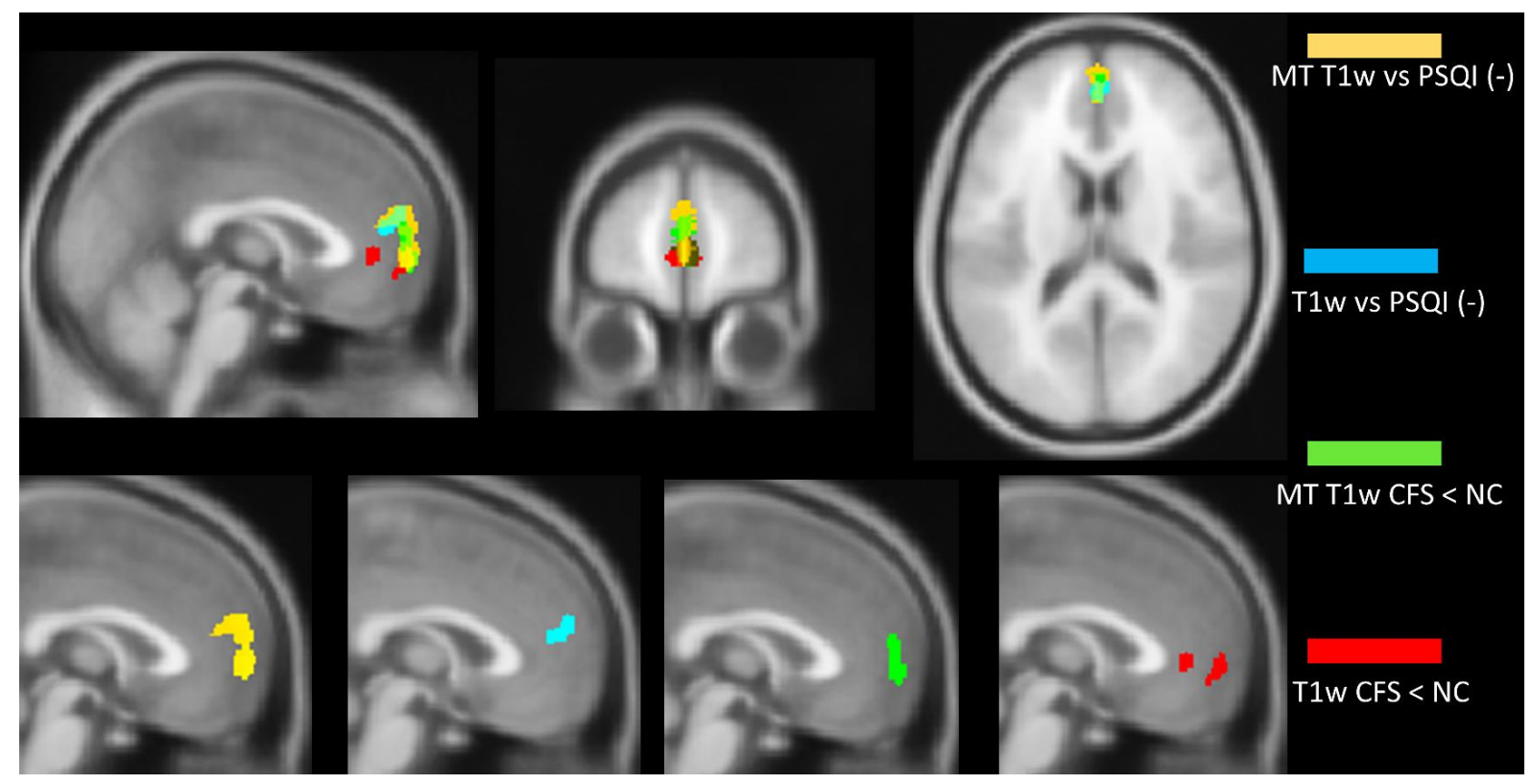

Fig 4. Four clusters in the medial prefrontal cortex from magnetization transfer $\mathbf{T 1}$ weighted spin echo (MT-T1w) and conventional T1 weighted spin echo (T1w) intensity analyses. 


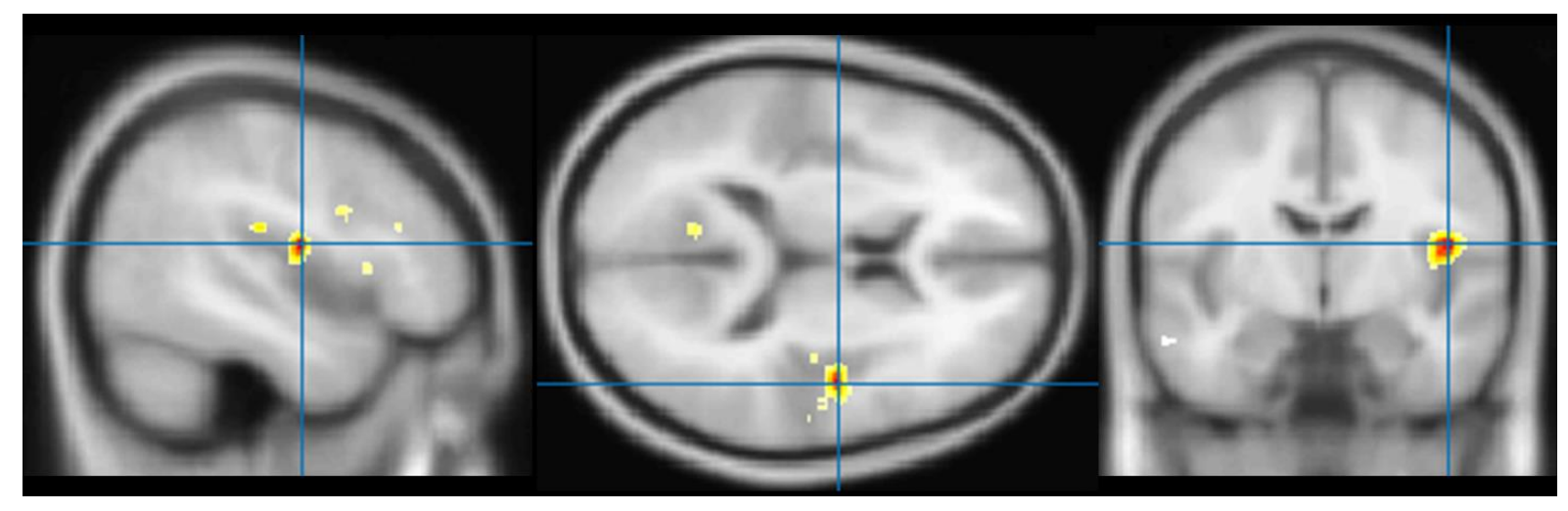

Fig 5. Results from voxel based regressions of the Chalder fatigue scale (CFQ) with T1 weighted spin echo (T1w) intensities. 